\title{
Design and Implementation of Traffic Monitoring System Based on Embedded Web Technology
}

\author{
Dr. Rajendra R. Dube ${ }^{1}$, Sushama S. Pawar ${ }^{2}$ \\ ${ }^{1}$ Head of Department of Electronics and Telecommunication Engineering, Walchand Institute of Technology, Solapur, India \\ ${ }^{2}$ PG Scholar, Department of Electronics Engineering, Walchand Institute of Technology, Solapur, India
}

\begin{abstract}
The ever increasing number of vehicles in most metropolitan cities around the world and the limitation in altering the transportation infrastructure, led to serious traffic congestion and an increase in the travelling time. we exploit the technologies such as the internet, to design an Traffic Monitoring System that can remotely monitor and control a network of traffic. The system is based on utilizing Embedded Web Servers (EWS) technology to design Traffic Monitoring System and also use embedded Linux operating system. Remote monitoring is realized to traffic information collection, monitoring traffic conditions, traffic control, information published and communication of traffic data by using combining EWS technology with Internet. The results indicate that the traffic monitoring based on embedded web technology can achieve the integration of a wide range of information collection The testing showed the traffic monitoring system based on embedded Web technology has the good real-time and high reliability and good scalability and antiinterference performance. Meanwhile, it has also laid a good foundation for the further study of a new type of traffic information collection system
\end{abstract}

Keywords: Raspberry Pi board ,Ultrasonic sensor,Embedded web server

\section{Introduction}

With rapid economic development, transportation has increasingly become an extremely important component in the national economy and daily life. So it is very essential to build a modern traffic control system in order to resolve the traffic congestion of roads and reduce accidents. Traffic for solving urban traffic management has become the people's consensus such as advanced and sophisticated video surveillance system as an important component of transportation for image acquisition, on-site snapshot, after taking of evidence and other important tasks. And video monitoring and traffic information transmission in this system plays an important role. Monitoring systems are usually installed on the expressway, traffic junctions, toll stations and other key places according to the actual needs of current traffic monitoring. All the information is integrated to the monitoring center.

At present, the traffic management monitoring systems are based on the PC as a host computer, and deploy dedicated monitoring configuration software. This method is not only costly, inefficient, but also troublesome for the system to update, and specialized training for management personnel, and restricted by space-time and geography. Moreover, some information cannot be shared for public information service. With the rise of the Internet technology, embedded Web technology goes into the mainstream at present, and Web server support the program running on an embedded device. The managers can manage and monitor situations of traffic through the Web browsers.

This presents a method that combines embedded WEB technology with Internet to implement remote traffic monitoring through Web Server applications solidified in embedded ARM processor. Therefore managerial personnel can have the remote real-time monitoring of traffic management through Web browsers without time and geographical constraints as shown in Figure 1. Timeconsuming effort of traditional local monitoring, as well as deficiencies in equipment maintenance, is effectively overcome and efficiency of traffic management is greatly improved. In order to classify information, embedded Web Server applications consist of different treatments of classification according to the types of information that are then properly displayed in the browsers. Attention is given especially for the effective separation of the confidential and public information.

\section{System Design}

\section{Architecture of Embedded Web Server}

A web server is a system which hosts a web site and provides services for any requesting clients. Fig. shows the design architecture of a typical EWS.

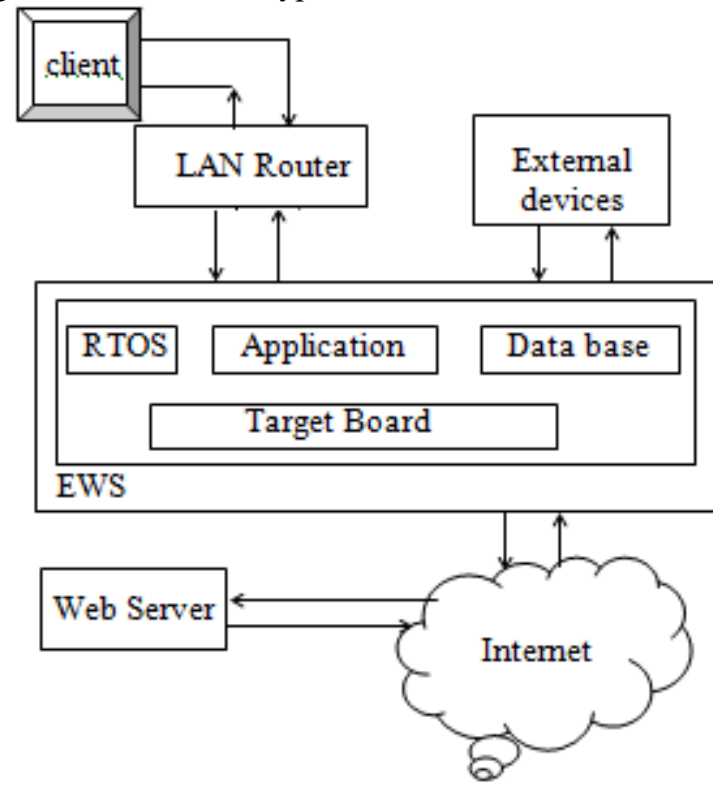

Figure 2: Architecture of a typical EWS 


\section{International Journal of Science and Research (IJSR) \\ ISSN (Online): 2319-7064}

Index Copernicus Value (2013): 6.14 | Impact Factor (2014): 5.611

The hardware used for embedded web server is ARM11 based board. The board has the html pages saved on it.The application runs in the form of tasks. Each user connecting to the server is treated as a task. To manage the users, connections, an operating system is required, that performs the operations in real time. The embedded web server is a powerful but small RTOS kernel. It is highly CPU independent and has been ported to numerous microprocessor platforms. To interact with the clients, the client has to send the data to them. In the embedded web server, web pages are selected as the media of interaction. The web pages are designed using HTML.

\section{Block Diagram}

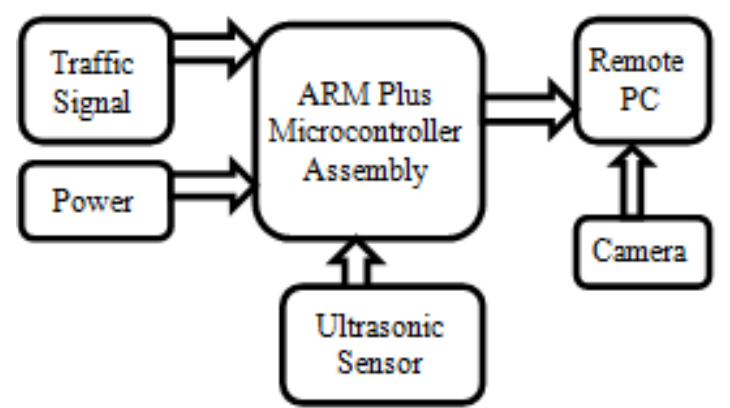

Figure 3: Basic Block Diagram

\section{Details of Hardware Required}

\section{Raspberry Pi}

The Raspberry Pi is a credit sized computer that plugs into your TV \& a keyboard.It is capable little computer which can be used in electronics projects \& for many of things that your desktop PC does, like spreadsheets, word processing \& games.Raspberry Pi requires 5 volt supply with minimum of 700-1000 mA current and it is powered through micro USB cable. It operates at $700 \mathrm{Mhz}$. C language is used to write the code into the raspberry pi. Raspberry $\mathrm{Pi}$ has a strong processing capacity because of using the ARM11 architecture and Linux-based system.In terms of control and interface, it has 8 GPIO, 1 UART, 1 I2C and 1 SPI, which are basically meet the control requirement. It has its own operating system.

\section{ARM7 Development Board}

LPC2148 Development Board is a powerful development platform based on LPC2148 ARM7TDMI microcontroller with $512 \mathrm{~K}$ on-chip memory. This board is powered by USB port and does not need external power supply. The on-chip USB controller provides direct high speed interface to a $\mathrm{PC} /$ laptop with speeds up to $12 \mathrm{Mb} / \mathrm{s}$. The UART boot loader eliminates need of an additional programmer and allows you to program using serial port. The ARM7 Development board has been designed for optimal use of the peripherals on-chip and interfaces on-board to create a variety of applications

\section{Ethernet:}

Ethernet is the networking technology used in many offices and homes to enable computers to communicate and share resources. Many Ethernet networks also connect to a router that provides access to the Internet .IEEE 802.3 supports a LAN standard originally developed by Xerox and later extended by a joint venture between Digital Equipment
Corporation, Intel Corporation and Xerox. This was called Ethernet.

\section{Ultrasonic sensor}

The GH-311 ultrasonic Motion sensor provides precise, noncontact distance measurements from about $2 \mathrm{~cm}$ ( 0.8 inches $)$ to 3 meters (3.3 yards). It has 3-pin male header used to supply power $(+5 \mathrm{~V})$, ground and signal. The header may be plugged directly into a breadboard or a standard 3-wire extension cable. It is very easy to connect to microcontrollers requiring only one I/O pin. The GH-311 sensor works by transmitting an ultrasonic (well above human hearing range) burst and providing an output pulse that corresponds to the time required for the burst echo to return to the sensor. By measuring the echo pulse width, the distance to target can easily be calculated.

\section{Webcam}

The Intex PC webcam with night vision is a versatile webcam packed with all the features that you could ever desire. Its sleek design and stylish look will sweep you off your feet. Not just the looks, but also the functionalities of this little thing make it the best among the rests.

\section{Software}

\section{RaspbianOS}

Raspbian is an unofficial port of Debian Wheezy armhf with compilation settings adjusted to produce code that uses "hardware floating point", the and will run on the Raspberry $\mathrm{Pi}$. The port is necessary because the official Debian Wheezy armhf release is compatible only with versions of the ARM architecture later than the one used on the Raspberry Pi (ARMv7-A CPUs and higher vs the Raspberry Pi's ARMv6 CPU).

\section{RTLinux}

Unlike Linux, RTLinux provides hard real-time capability. It has a hybrid kernel architecture with a small real-time kernel coexists with the Linux kernel running as the lowest priority task. This combination allows RTLinux to provide highly optimized, time-shared services in parallel with the realtime, predictable, and low-latency execution. Besides this unique feature, RTLinux is freely available to the public. As more development tools are geared towards RTLinux, it will become a dominant player in the embedded market.

\section{System Function and Testing}

The embedded Web traffic monitoring system is based on embedded Web technology as the core. Meanwhile, it is combined with traffic information acquisition, traffic surveillance, traffic control, information publication and other traffic control functions. Then the traffic data are collected, stored, managed, transmitted, analyzed, and displayed. The traffic managers or decision makers are provided with these data for decision making and management on the traffic situation.

For example, when a vehicle runs the red light, cameradetects the vehicle through the ultrasonic sensor; its detector is triggered, at the same time the signal controller issues a "red light". When both conditions are matching, an 


\section{International Journal of Science and Research (IJSR) \\ ISSN (Online): 2319-7064}

Index Copernicus Value (2013): 6.14 | Impact Factor (2014): 5.611

image of the vehicle, with the relevant monitoring information is taken as illegal driving. For another example, when a vehicle runs the green signal, count the number of vehicles passes. According to the number of vehicles passes define the density of vehicle i.e.Low,Medium,High. If density indicates high then increase the time automatically Also at green signal the speed of the vehicle is measured. If the speed exceeds the setting, it will direct the camera to obtain the relevant speeding monitoring information with image of that vehicle. With heterogeneous network seamlessly connected with embedded gateway design as well as the realization of key technologies, these traffic monitoring information are integrated to the EWS through a serial port.

On-site monitoring traffic information is sent to the web browsers via a EWS. So wherever the monitoring personnel are, the target region can be monitored and managed as long as it is connected to the Internet network Diagram of system function and Traffic information with web browser shown in following figure.

Traffic signal

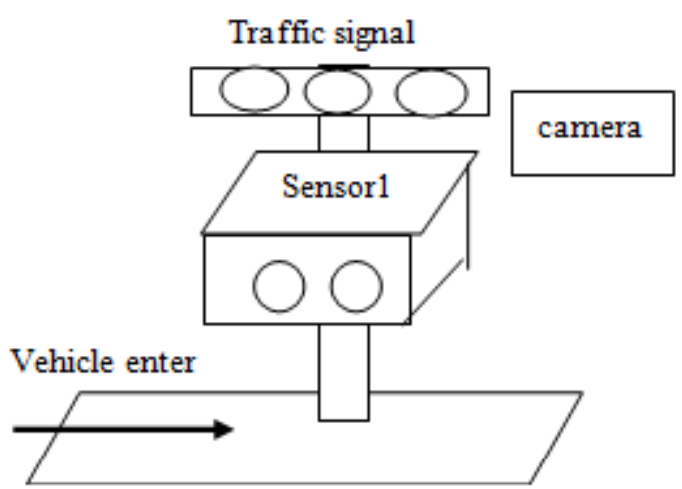

Figure 4: For Vehicle Detection

\section{Camera}
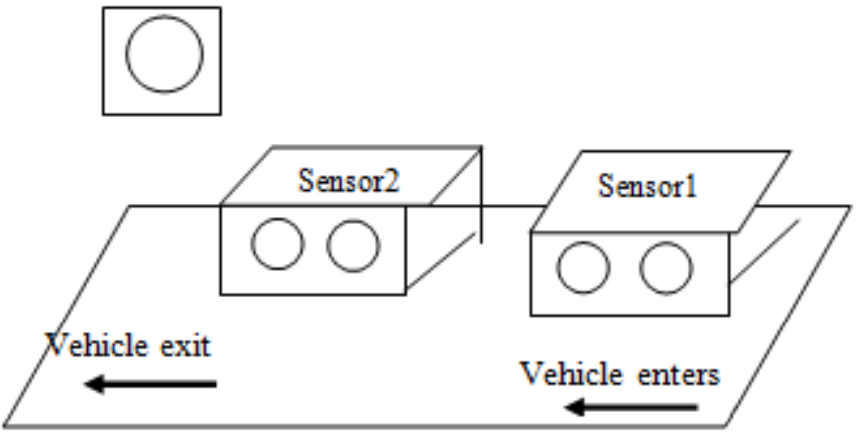

Figure 5: For Speed Measurement
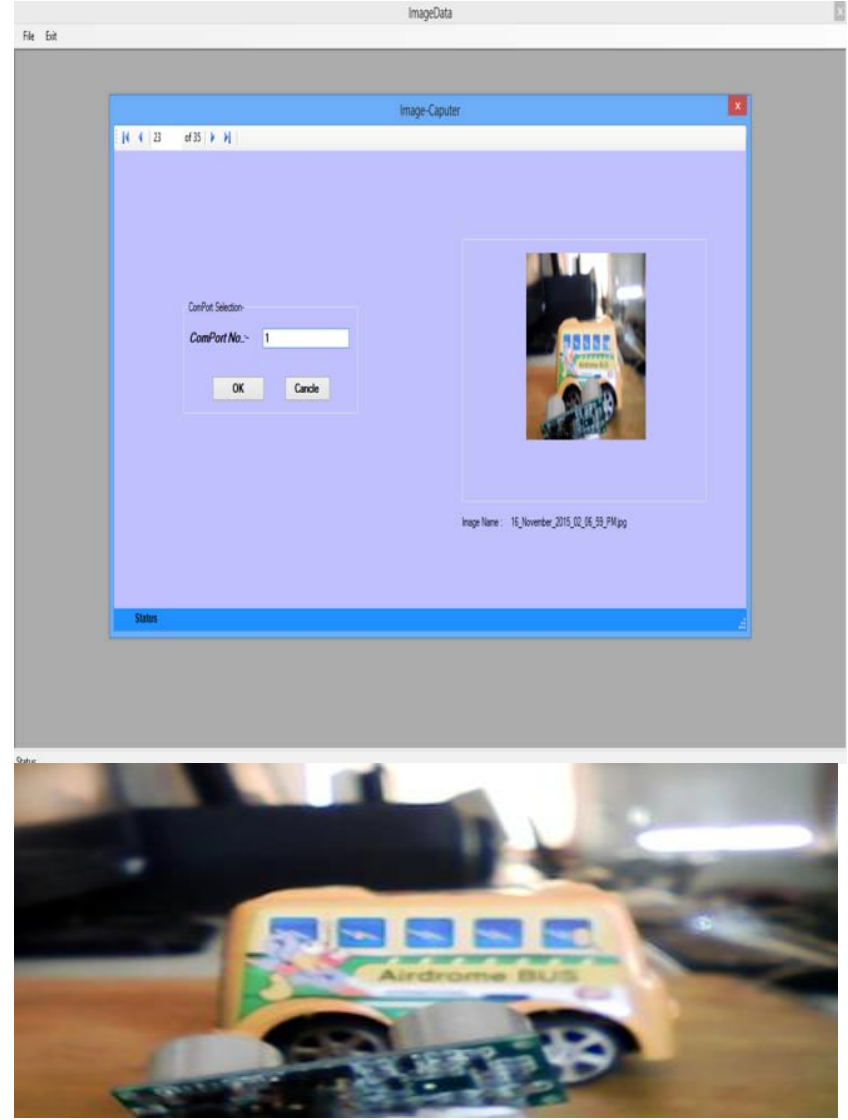

Figure 6: Captured Image of Vehicle
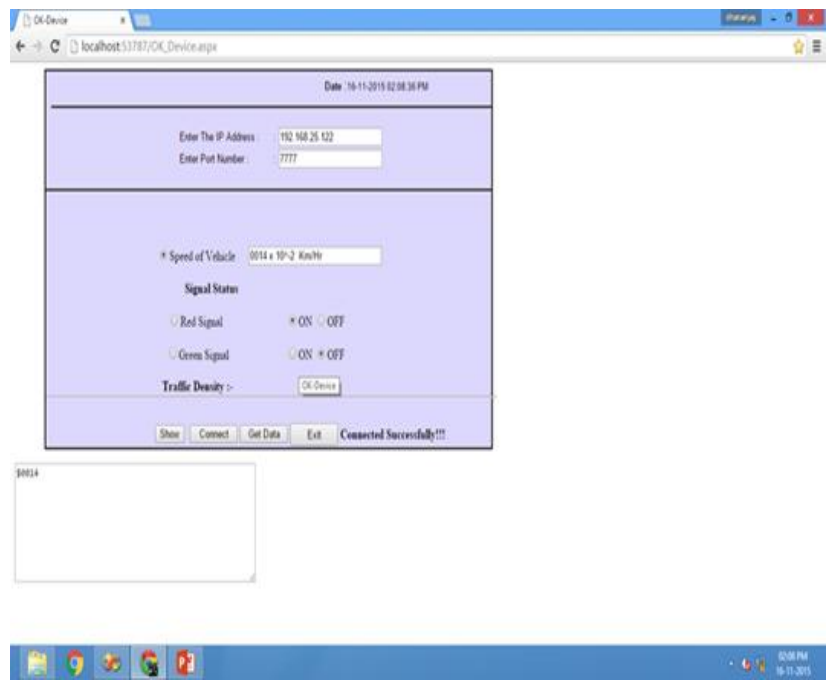

Figure 7: Speed of Vehicle

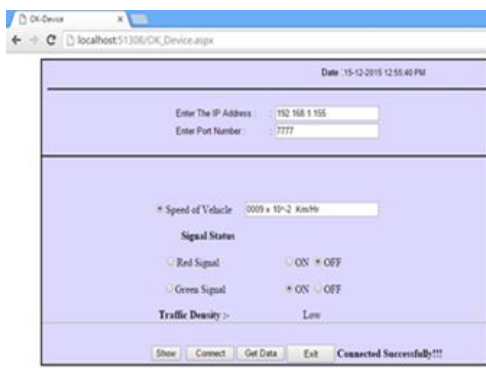




\section{International Journal of Science and Research (IJSR) \\ ISSN (Online): 2319-7064}

Index Copernicus Value (2013): 6.14 | Impact Factor (2014): 5.611

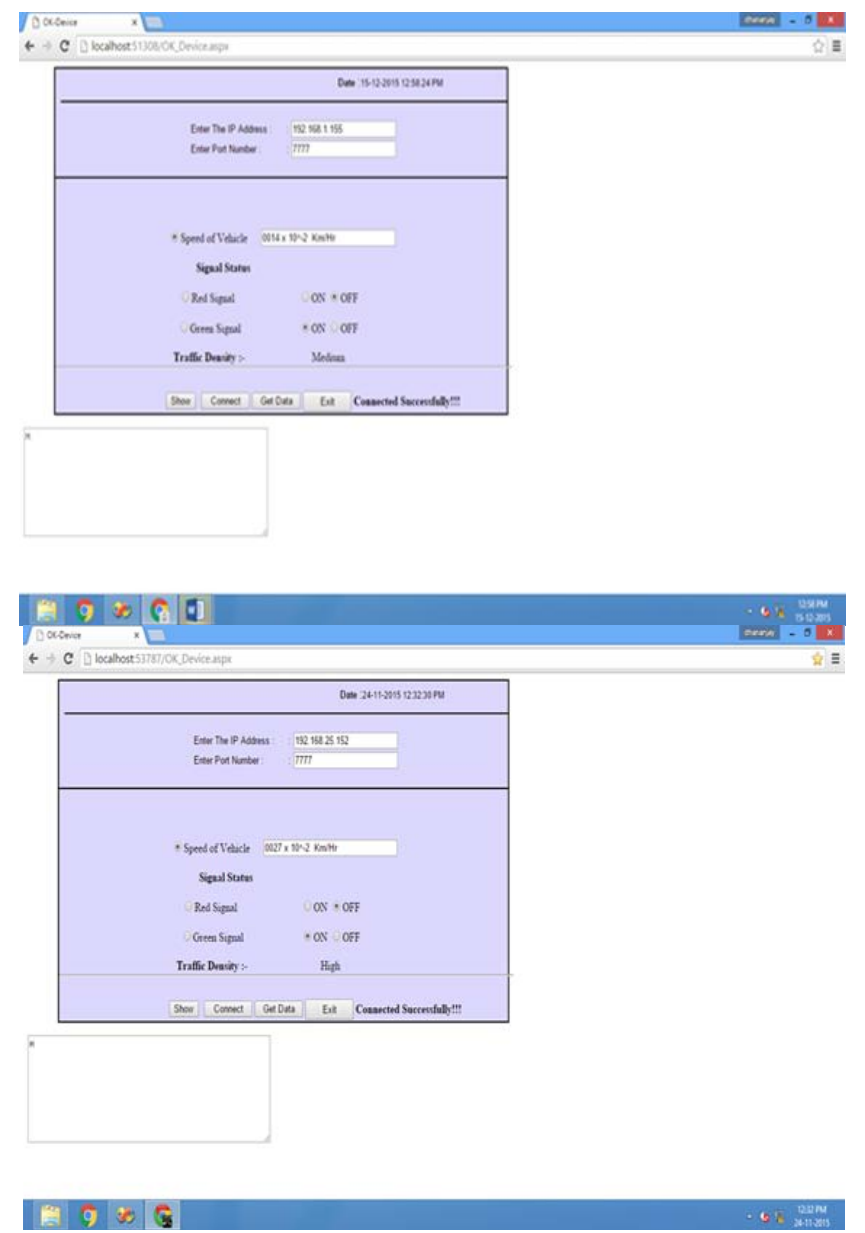

Figure 8: Densities\& Speed of Vehicle

The table below shows the results in tabular form

\begin{tabular}{|c|c|c|}
\hline Date \& time & Speed of vehicle & Density \\
\hline $15-12-201512: 55: 40 \mathrm{pm}$ & $0009^{*} 10^{\wedge}-2 \mathrm{~km} / \mathrm{hr}$ & Low \\
\hline $15-12-201512: 58: 24 \mathrm{pm}$ & $0014^{*} 10^{\wedge}-2 \mathrm{~km} / \mathrm{hr}$ & Medium \\
\hline $24-11-201512: 32: 30 \mathrm{pm}$ & $0027^{*} 10^{\wedge}-2 \mathrm{~km} / \mathrm{hr}$ & High \\
\hline
\end{tabular}

Figure 6.4: Speed \& Density of vehicle in tabular form

\section{Conclusion}

The traffic monitoring system based on embedded Web technology possesses the low power consumption, high integration, real-time efficiency, software solid-state, and easy scalability. Using this technology monitoring the traffic easily with traffic information. If density is high then the time increases automatically hence by using this control the density of traffic.

\section{References}

[1] Han XiaoTao,Yin XiangGen, Zhang Zhe, LI Wei, "Review of embedded web server technology and its application in power system" [J] Power System Technology 2003,(5): 58-62

[2] Fu BaoChuan, Ban JianMin, "Design of remote monitoring system based on Web embedded" [J].Control \& Automation(Embedded and SOC),2005,21,7-2:58-60

[3] Sing Y., Sinem C., Baris D., Sumitra G., Chin-Woo T., Pravin V., "Traffic measurement and vehicle classification with a single magnetic sensor", Journal of the Transportation Research Board, February, 2006.

[4] Igor Klimchynski, "Extensible Embedded Web Server Architecture for Internet-Based Data Acquisition and Control," IEEE Sensors Journal, Vol. 6, No. 3, June 2006.

[5] LI Yong," Application and realization of CGI in embedded WEB server"'J].Microcomputer Information, 2008,(30):110- 111.

[6] Zong Chang-Fu, Yang Xiao, Wang Chang, et al, "Driving intentions identification and behaviors prediction in car lane change" $[\mathrm{J}]$. Journal of Jilin University (Engineering and Technology Edition), 2009, 39: 27-32.

[7] V.Billy Rakesh Roy1, Sanket Dessai1, and S. G.Shiva Prasad Yadav 1 "Design and Development of ARM Processor Based Web Server' International Journal of Recent Trends in Engineering, Vol. 1, No. 4, May 2009

[8] Yakun Liu Xiaodong Cheng "Design and Implementation of Embedded Web Server Based on ARM and Linux" 978-1-4244-7656-511 01\$26.00 (C) 2010 IEEE

[9] B. Srinivas Raja and G. Srinivas Babu "Design of Web based Remote Embedded Monitoring system",Cgopalax -International Journal of Technology And Engineering System(IJTES): Jan -March 2011- Vol.2.No.2

[10] Adajania, V., Agarwal, M Dandekar, S.andKaria, D.C.,"EmbeddedWeb Server Application Based Automation and Monitoring System," International Conference on Signal Processing, Communication, Computing and NetworkingTechnologies, pp.634-637, July 2011.

[11] ServerSoumya Sunny P1, Roopa .M , “Data Acquisition and Control System Using Embedded Web server,'International Journal of Engineering Trends and Technology- Volume3Issue3- 2012

[12]Lu Jian-feng, Wang Chun-yi, Hu Jie "A High Performance Data Storage Method for EmbeddedLinux Real-time Database in Power Systems" Energy Procedia 16 (2012) $883-888$

[13] Taner Arsan, Alp Parkan and Hakkı Konu ,"Design and implementation of remotelyManaged embedded digital signage system" International Journal of Computer Science, Engineering and Applications (IJCSEA) Vol.4, No.3, June 2014

[14] http://www.raspberrypi.org

[15] www.opensource.org 